\section{Neuro-ophthalmological manifestations after \\ intramuscular medroxyprogesterone: a forme fruste of idiopathic intracranial hypertension?}

Mandreker Bahall,1,2 Antonio Jose Reyes,1

Kanterpersad Ramcharan, ${ }^{3}$

Nadeem Hosein, ${ }^{3}$ Karan Seegobin, ${ }^{1}$

Krishni Bahall, ${ }^{1}$ Hiranyadeva Sharma, ${ }^{1}$

Stephanie Dhansingh, ${ }^{1}$ Amanda Mahabir ${ }^{1}$

1Department of General Medicine, San

Fernando General Hospital; 2Department

of Clinical Medical Sciences, University of

the West Indies, St. Augustine;

3Neurology Unit, San Fernando Teaching

Hospital, University of West Indies, San

Fernando, Trinidad and Tobago

\begin{abstract}
We report a case of a 22-year-old female student nurse who presented to hospital with an acute neuro-ophthalmological syndrome characterized by papilledema, ataxia, ophthalmoplegia and headache after a single first time use of $150 \mathrm{mg}$ medroxyprogesterone intramuscular injection. Clinical, laboratory, radiological and ophthalmological investigations were in keeping with the diagnosis of idiopathic intracranial hypertension but lumbar puncture did not show a raised cerebrospinal fluid pressure suggesting a forme fruste of this entity. Her neuro-ophthalmological clinical features responded well to acetazolamide and diagnostic/therapeutic lumbar puncture. Full recovery was achieved three months after medroxyprogesterone usage. Health care providers must be aware of this adverse drug reaction.
\end{abstract}

\section{Introduction}

Idiopathic intracranial hypertension (IIH), associated with medroxyprogesterone use, has been seldom documented. ${ }^{1}$ Also atypical presentations that partially fulfill the classic definition of intracranial hypertension by the modified Dandy criteria (forme fruste variant) are also rarely reported. Despite posing a diagnostic challenge, such atypical cases can be identified and managed with a positive outcome by the implementation of clinical assessments based on a less rigid modified Dandy criteria. ${ }^{2}$ To the best of our knowledge, papilledema, ataxia, ophthalmoplegia and headache in the same patient after a single medroxyproges- terone dosage has not been described previously in the medical literature.

\section{Case Report}

A 22-year-old non vegetarian AfroTrinidadian female, previously well, active and working as a student nurse, presented to the emergency department of hospital with severe left sided frontal headaches and vomiting over two days. Her symptoms were associated with fever, unsteady gait and dizziness. She had no history of head trauma or use of recreational drugs, alcohol, smoking or exposure to chemicals at work. However, the patient reported a first time use of a single intramuscular dosage of $150 \mathrm{mg}$ of medroxyprogesterone for contraceptive purpose two weeks before date of admission to hospital. She had no previous medical or surgical problems. Patient's family history was unremarkable. On physical examination the patient was in no cardiorespiratory distress with a Glasgow Coma Scale of 15/15. She was oriented to time, place and person. Blood pressure was 113/69 mm of $\mathrm{Hg}$, heart rate was 106 beats per minute regular, respiratory rate was 18 breaths per minute, body temperature was $36.5^{\circ} \mathrm{C}$, oxygen saturation of $100 \%$ on room air and patient's body mass index was 25 . Visual acuity, visual field, pupillary reflexes, eye movements and other cranial nerves examination were initially normal. Tone and power in upper and lower limbs were normal. Deep tendon reflexes were equal and symmetrical +++ at ankles, knees, biceps and triceps bilaterally. Plantar response showed positive Babinski's sign bilaterally. Sensory, vibration and proprioception tests were normal. Kernig's and Brudzinski's signs were negative. Romberg's test was positive and associated with wide based stance and ataxic gait. The patient showed poor coordination of voluntary movements during rapid alternate movements, finger to nose and heel to shin tests. On the second day of admission to hospital, the patient developed blurred vision and diplopia bilaterally. Fundoscopic examination showed bilateral papilloedema. Full blood count report revealed that hemoglobin was $129 \mathrm{~g} / \mathrm{L}$ (female 120-156 $\mathrm{g} / \mathrm{L})$, platelet count was $187 \times 10^{9}$ platelets $/ \mathrm{L}$ (150-350 $\times 10^{9}$ platelets/L), white cell count was $8.4 \times 109 / \mathrm{L}\left(4.5-11.0 \times 10^{9} / \mathrm{L}\right)$, mean red blood cell volume was $88.8 \mathrm{fL}(80-100 \mathrm{fL})$, hematocrit was 0.38 (female 0.36-0.46). A blood film showed normochromic and normocytic red blood cells. Renal function tests, liver function tests, thyroid function tests, C3, C4, and electrolytes were all within normal limits. $\mathrm{C}$ reactive protein was 1.0 $\mathrm{mg} / \mathrm{L}$. Urine beta human chorionic gonadotropin, HIV rapid, Venereal Disease Research Laboratory, Mantoux and Ebstein barr antibody tests were all negative. Autoimmune
Correspondence: Mandreker Bahall, Department of General Medicine, San Fernando General Hospital, House \#57 LP 62, Calcutta Road Number 3, McBean, Couva, Trinidad, Trinidad and Tobago. Tel: +1.8687636608 - Fax: +1.8686790816 . E-mail: vmandrakes@hotmail.com

Key words: Ataxia; papilledema; medroxyprogesterone; modified Dandy criteria; forme fruste.

Acknowledgements: the authors would like to thank Dr. Vish Ramnath (Consultant Ophthalmologist) for performing ophthalmological examinations, visual perimetry and optical coherence tomography.

Contributions: the authors contributed equally.

Conflict of interest: the authors declare no potential conflict of interest.

Received for publication: 2 August 2015.

Revision received: 25 January 2016.

Accepted for publication: 8 August 2016.

This work is licensed under a Creative Commons Attribution NonCommercial 4.0 License (CC BYNC 4.0).

(C) Copyright M. Bahall et al., 2016

Licensee PAGEPress, Italy

Neurology International 2016; 8:6132

doi:10.4081/ni.2016.6132

and vasculitic screen: antinuclear antibodies, DS-DNA, atrial natriuretic factor, SS-B, Scleroderma-70, PM-SCL, J0-1, centromere autoantigen $\mathrm{B}$, proliferating cell nuclear antigen, antinucleosome antibodies, histone antibodies, antiribosomal $\mathrm{P}$ protein antibodies, AMA-M2, lupus anticoagulant, P-antineutrophil cytoplasmic antibodies (ANCA), and C-ANCA were all negative. Serum vitamin B12 was 2000 $\mathrm{pg} / \mathrm{mL}$ (211- $946 \mathrm{pg} / \mathrm{mL})$. Serum methylmalonic acid was $427 \mathrm{nmol} / \mathrm{L}(0-378 \mathrm{nmol} / \mathrm{L})$, serum homocysteine was $14.2 \mathrm{umol} / \mathrm{L} \quad(3.4-20.4$ umol/L), parietal cell antibodies were less than $2 \mathrm{RU} / \mathrm{mL}$ (Negative when less than 20) and intrinsic factor antibodies were less than 2 RU/mL (Negative when less than 20). Tissue transglutaminase antibodies (IgA, IgG) and endomysial and Anti-Saccharomyces cerevisiae antibodies were negative. A lumbar puncture done 8 days after admission showed an opening cerebrospinal fluid (CSF) pressure of $13 \mathrm{~cm}$ of $\mathrm{H}_{2} \mathrm{O}$, clear CSF fluid with normal cell count, protein and glucose. CSF culture showed no bacterial growth. CSF displayed 3 unmatched oligoclonal IgG bands and one matched oligoclonal IgG band using isoelectric focusing and immunoblotting methodology.

A brain computerized axial tomography scan was normal. Brain magnetic resonance imaging (MRI) scan, magnetic resonance angiography scan and magnetic resonance venography scan were all normal (Figure 1). MRI scan of 
the spine was normal.Visual perimetry showed enlargement of blind spot in both eyes. Optical coherence tomography of both eyes was normal. The patient was diagnosed then as suffering from possible IIH induced by medroxyprogesterone. She was treated with oral acetazolamide $300 \mathrm{mg}$ three times daily in addition to a therapeutic lumbar puncture, following which there was complete resolution of patient's headaches, vomiting and blurred vision. Due to rapid improvement repeat lumbar puncture was considered unnecessary. She had minimal improvement of gait but Romberg's test and Babinski became negative. The patient was discharged after 15 days in hospital with continued physiotherapy, medical outpatient clinical follow up and advised on cessation of medroxyprogesterone. She developed complete ophthalmological and neurological recovery three months after administration of medroxyprogesterone. She has since returned to her job and performing normal duties as a student nurse. Brain MRI scan sequences including fluid inversion recovery images performed one year later remained the same with normal third, laterals and fourth ventricles without transependymal changes.

\section{Discussion}

IIH typically presents with headache, pulsatile tinnitus, dizziness, nausea, vomiting, visual disturbance, papilledema and elevated CSF pressures. However these symptoms and signs are often not all present together, and they can vary with time in any given patient.1,2 The current modified Dandy criteria used for the diagnosis are: i) signs and symptoms of increased intracranial pressure; ii) no other neurological abnormalities or impaired level of consciousness; iii) elevated CSF opening pressure with normal CSF composition; iv) a neuroimaging study that shows no etiology for increased intracranial pressure; v) no other cause for intracranial hypertension found.2,3 Our patient did not completely meet the modified Dandy criteria for IIH as CSF pressure was normal. Diurnal variations in CSF pressure, however, have been described in the literature thus this fact does not exclude the diagnosis of intracranial hypertension in our patient. ${ }^{3}$ Though difficult to rule out, our case does not appear to be normal pressure hydrocephalus as the MRI study did not show hydrocephalus or transependymal changes suggestive of edema. No cause for obstruction to CSF flow was seen on MRI. Of concern is the normal optical coherence tomography (OCT) in the presence of papilledema. However this may result because of poor quality images with false readings and the possibility of false negatives. Currently, OCT does not replace visual field testing and

\section{fundus examination. 4,5}

Several reports have associated IIH with exogenous and endogenous estrogens, especially those containing oral ethinyl estradiol and progesterone only injectable. Many associations of IIH with various medications including medroxyprogesterone have not been well substantiated.1,3,6-8 To the best of our knowledge, only two cases have been reported in the literature separately: a 29 and a 23 year old woman who developed IIH 8 weeks after a single injection of $150 \mathrm{mg}$ of medroxyprogesterone.1,7,9 Our case may represent a forme fruste of this entity. Between January 2004 and April 2015, a total of 9972 post market medroxyprogesterone drug adverse event reaction reports were made to the Food and Drug Administration (FDA). Among them, 8 people reported ataxia to the FDA. More than 250 different drugs were reported to the FDA as causing ataxia. 10 Nagel and colleagues, reported that $33 \%$ of women $(15 / 45)$ treated with 1500 mg orally daily of medroxyprogesterone for advanced breast cancer developed ataxia.11 Some reported cases of reversible ataxia have been associated with hypomagnesaemia, hyponatremia, metronidazole and thyrotoxicosis.12-15 In Miller Fisher syndrome (ataxia, arreflexia and ophthalmoplegia) IIH have been reported but this syndrome has not been associated with medroxyprogesterone. 16

There was a close temporal relationship
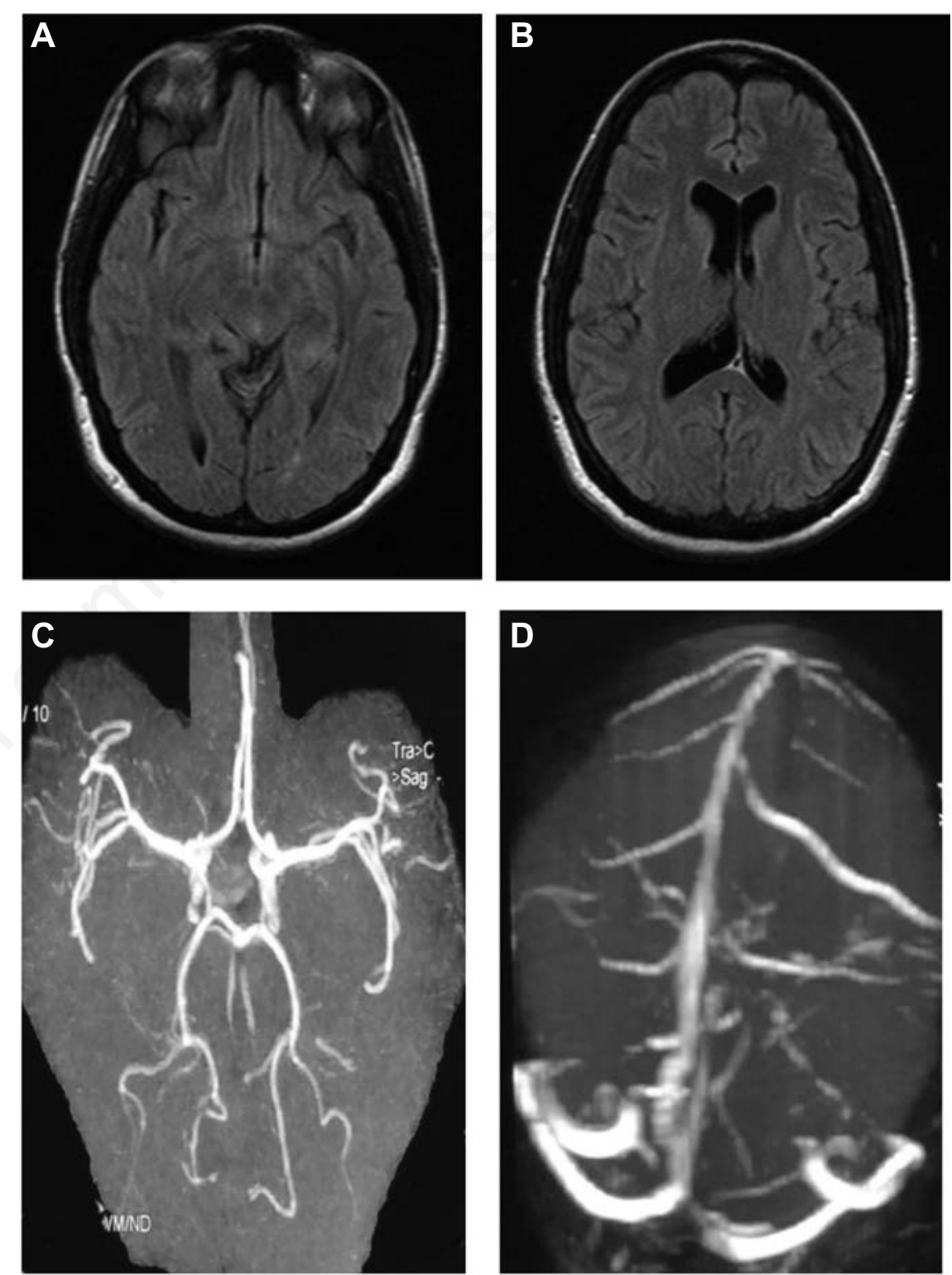

Figure 1. A,B) Axial flair magnetic resonance sequence images of the brain showing normal ventricles without transependymal changes; $C$ ) magnetic resonance angiography scan of Circle of Willis arteries and its main branches; D) magnetic resonance venography scan section, demonstrating normal studies. 
between the administration of the contraceptive injection and the manifestation of symptoms and signs of IIH with ataxia in our adult patient. The temporal relationship of two weeks seen in our patient was different to the temporal relationship of eight weeks reported in the medical literature in the other two cases. ${ }^{1,7}$ The serum level from a recommended dose of medroxyprogesterone of $150 \mathrm{mg}$ intramuscularly usually increases over 3 weeks to reach peak plasma concentrations between 1 and $7 \mathrm{ng} / \mathrm{mL}$. The levels then exponentially decrease until they become undetectable ( $<100 \mathrm{pg} / \mathrm{mL}$ ) between 120 to 200 days following injection. ${ }^{7}$ The exact pathogenesis of opthalmoplegia, headaches and papilledema with or without ataxia induced by progestins remains unclear. The close temporal relationship between the administration of the contraceptive and the manifestation of the neuroophthalmological syndrome and complete resolution of the clinical manifestations after three months when the drug would have been cleared are suggestive of a causal relationship.

\section{Conclusions}

This case demonstrated that medroxyprogesterone induced papilledema, ataxia, ophthalmoplegia and headache that completely reversed by three months most probably represented a forme fruste of IIH in keeping with previous reported cases of partial clinical expression of this illness. This drug given intramuscularly every three months is com- monly used by many women as a form of birth control and clinicians need to be vigilant in recognizing this adverse event.

\section{References}

1. Faculty of Sexual and Reproductive Healthcare Clinical Effectiveness Unit of The Royal College of Obstetricians and Gynecologists. Are progestogen only contraceptives implicated in the cause of idiopathic intracranial hypertension? Available from: https:/www.fsrh.org/documents/ceu-edme0092-may2015/

2. Shaw GY, Million SK. Benign intracranial hypertension: a diagnostic dilemma. Case Rep Otolaryngol 2012;2012:814696.

3 . Wall M. Idiopathic intracranial hypertension. Neurol Clin 2010;28:593-617.

4. Bidot S, Vasseur V, Vignal-Clermont C. Optical coherence tomography and intracranial hypertension. J Fr Ophtalmol 2013;36:277-85.

5. Bassi ST, Mohana KP. Optical coherence tomography in papilledema and pseudopapilledema with and without optic nerve head drusen. Indian $\mathrm{J}$ Ophthalmol 2014;62:1146-51.

6. Salmon ML, Winkleman JZ, Gay AJ. Neuroophthalmic sequelae in users of oral contraceptives. JAMA 1968;206:85-91.

7. Chan JW. Idiopathic intracranial hypertension associated with depot medroxyprogesterone. Eye 2006;20:1396-7.

8. Sheehan JP. Hormone replacement treat- ment and benign intracranial hypertension. Br Med J (Clin Res Ed) 1982;284: 1675-6.

9. Bahemuka M. Benign intracranial hypertension associated with the use of depoprovera (depot medroxyprogesterone): a case report. East Afr Med J 1981;58:140-1.

10. eHealthMe Personalized Health Information. Review: could Depo-provera cause ataxia? Available from: http://www.ehealthme.com/ds/depoprovera/ataxia

11. Nagel GA, Wander HE, Biossey HC. Phase II study of aminoglutethimide and medroxyprogesterone acetate in the treatment of patients with advanced breast cancer. Cancer Res 1982;42:3442s-4s.

12. Boulos MI, Shoamanesh A, Aviv R, et al. Severe hypomagnesemia associated with reversible subacute ataxia and cerebellar hyperintensities on MRI. Neurologist 2012;18:223-5.

13. Kelsey SM, Williams AC, Corbin D. Hyponatraemia as a cause of reversible ataxia. Br Med J (Clin Res Ed) 1986; 293:1346.

14. Rama PS, Suresh G, Prakash PS, D'sa IP. Reversible cerebellar ataxia with thyrotoxicosis: an autoimmune brain disease in remission due to Graves' disease. Indian J Endocrinol Metab 2013;17:519-20.

15. Sarna JR, Brownell AKW, Furtado S. Reversible cerebellar syndrome caused by metronidazole. CMAJ 2009;181:611-3.

16. Mewasingh LD, Sékhara T, Dachy B, et al. Benign intracranial hypertension: atypical presentation of Miller Fisher syndrome? Pediatr Neurol 2002;26:228-30. 